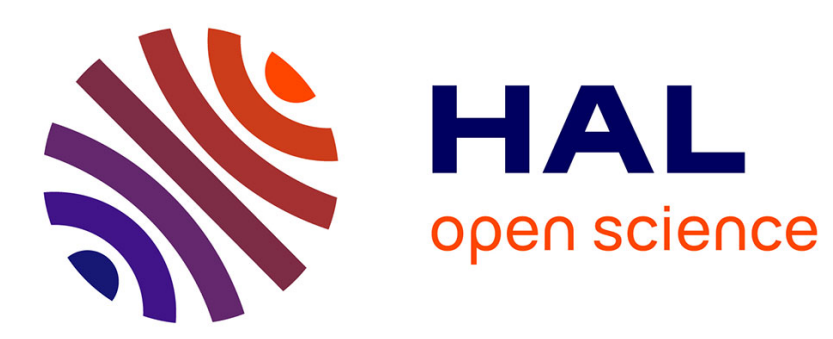

\title{
Détermination électrochimique de l'épaisseur des biofilms dans les circuits d'eau
}

\author{
C. Forêt, N. Merlet, Bernard Tribollet, G. Chaussec, B. Legube
}

\section{To cite this version:}

C. Forêt, N. Merlet, Bernard Tribollet, G. Chaussec, B. Legube. Détermination électrochimique de l'épaisseur des biofilms dans les circuits d'eau. Matériaux \& Techniques, 2006, 94, pp.467 - 476. 10.1051/mattech:2007021 . hal-01729171

\section{HAL Id: hal-01729171 \\ https://hal.sorbonne-universite.fr/hal-01729171}

Submitted on 12 Mar 2018

HAL is a multi-disciplinary open access archive for the deposit and dissemination of scientific research documents, whether they are published or not. The documents may come from teaching and research institutions in France or abroad, or from public or private research centers.
L'archive ouverte pluridisciplinaire HAL, est destinée au dépôt et à la diffusion de documents scientifiques de niveau recherche, publiés ou non, émanant des établissements d'enseignement et de recherche français ou étrangers, des laboratoires publics ou privés. 


\section{Détermination électrochimique de l'épaisseur des biofilms dans les circuits d'eau}

\section{Forêt ${ }^{1}$, N. Merlet ${ }^{2}$, B. Tribollet ${ }^{3}$, G. Chaussec ${ }^{1}$ et B. Legube ${ }^{2}$}

1 HENKEL CONCORDE, 43 route de Ruaudin, 72230 Arnage, France e-mail : Guenole.Chaussec@fr.henkel.com ; Christophe.Foret@fr.henkel.com

2 Laboratoire de Chimie de l'Eau et de l'Environnement, UMR CNRS 6008, École Supérieure d'Ingénieurs de Poitiers, Université de Poitiers, 40 avenue du recteur Pineau, 86022 Poitiers Cedex, France e-mail : nicole.merlet@esip.univ-poitiers.fr; bernard.legube@esip.univ-poitiers.fr

3 Laboratoire Interfaces et Systèmes Électrochimiques, UPR CNRS 15, Université Pierre et Marie Curie, Case courrier 133, 4 place Jussieu, 75252 Paris Cedex 05, France

e-mail : bt@ccr.jussieu.fr

\section{Mots-clés :}

Biofilms; légionelle; circuits de refroidissement; épaisseur; évaluation du risque

Key words:

Biofilm; legionella; cooling systems; thickness; risk assessment

\begin{abstract}
Résumé - La croissance de biofilms sur les parois des réseaux et des circuits d'eaux est un problème récurrent en terme de santé publique. Les légionelles qui se développent dans les réseaux d'eaux chaudes et les tours aéroréfrigérantes ont été à l'origine de plus de 1000 cas annuels de légionellose en France au cours des 3 dernières années, la létalité étant d'environ $14 \%$. Pour maîtriser ce développement bactérien, il est nécessaire de posséder une meilleure compréhension des biofilms, de leur croissance et de leur composition. Dans ce but, trois années de travail de collaboration entre la société Henkel Concorde et 2 équipes CNRS (UMR 6008 et UPR 15) ont permis de développer un outil de détermination de l'épaisseur moyenne du biofilm. Sur des unités pilote en laboratoire et sur site industriel, cette mesure de l'épaisseur du biofilm s'est révélée suffisamment précise et sensible pour suivre l'évolution de la formation d'un biofilm dans un circuit d'eau et pour déterminer l'efficacité des traitements appliqués. L'utilisation de cet outil de surveillance a permis d'optimiser les traitements appliqués (nature, dose, fréquence d'injection) et conduit à une réduction significative des biomasses fixées, au maintien de la propreté des circuits et donc à une maîtrise accrue du risque légionelle.
\end{abstract}

\begin{abstract}
Electrochemical determination of biofilm thickness in water systems. Biofilms are growing on the internal wall of the water pipelines, this is a constant problem of health risk. The legionella which proliferate in the warm waters networks and in cooling towers, are at the origin of more than 1000 cases of Legionnaire's disease per year in France during the last three years, the death rate being about $14 \%$. To control this bacterial development, a better understanding of biofilms ecology is necessary, in particular their growth and their composition. In this purpose, three working years of collaboration between the company Henkel Concorde and 2 CNRS research teams (UMR 6008 and UPR 15) allowed to develop a tool able to measure the biofilm thickness. On laboratory pilots and in industrial site, this biofilm thickness measurement showed a very acceptable reliability and sensitivity to follow the behaviour of a biofilm in a water circuit and to evaluate the efficiency of the treatments (dispersing, biocide) which are applied. The use of this tool allows to optimize the applied treatments (nature, dose, frequency of injection) and leads to a strong reduction of the sessile biomass. Thanks to this sensor the cleanliness of water circuits could be maintained with a better efficiency and thus a greater control of the legionella risk could be obtained.
\end{abstract}

es parois internes de divers réseaux et circuits d'eau en milieu urbain ou industriel sont rapidement colonisées par des bactéries. Cette formation récurrente de biofilms est parfois à l'origine de la dégradation de la qualité des eaux et de problèmes de contamination bactériologique des eaux transportées (coliformes dans les réseaux d'eaux potables, Pseudomonas ou légionelles dans les réseaux d'eaux chaudes sanitaires ou les circuits d'eau des établissements recevant du public, légionelles dans les circuits de refroidissement ou les tours aéroréfrigérantes, libres ou associées à des protozoaires tels que les amibes et/ou ciliés).

Les biofilms sont composés d'un consortium très complexe de microorganismes [1], de substances polymériques extracellulaires [2], mais aussi 
d'autres molécules organiques, telles que les substances humiques $[2,3]$. Il a été récemment montré que les biofilms comportaient une composante minérale forte, avec la présence de dépôts minéraux associés à des biofilms d'eau naturelle $[4,5]$. Cette organisation en biofilm procure aux microorganismes des conditions adéquates pour leur multiplication et leur survie, et augmente leur résistance à la désinfection.

Pour limiter les risques de proliférations bactériennes dans les réseaux et respecter les prescriptions réglementaires, les établissements concernés doivent mettre en place des procédures adaptées pour assurer l'entretien et la maintenance des installations. Concernant la légionellose (1527 cas déclarés en France en 2005, avec une létalité de $11 \%$ ), les principaux réservoirs identifiés sont les réseaux d'eaux chaudes sanitaires et les circuits de refroidissement équipés de tours aéroréfrigérantes. Plus de 7000 circuits et 14000 tours aéroréfrigérantes ont été recensés fin 2005 et le renforcement de la réglementation, engagé fin 2004 (décret No. 2004-1331 du $1^{\text {er }}$ décembre 2004), oblige ces établissements à mettre en place des moyens de prévention.

Ces traitements préventifs passent par des nettoyages et des désinfections chimiques en continu ou ponctuellement, et l'efficacité de ces traitements est généralement évaluée par la teneur en légionelles dans les eaux des circuits, soit en déterminant la cultivabilité des légionelles [6], soit par des méthodes de biologie moléculaire [7]. Mais le délai de réponse de ces outils analytiques est au minimum de 24 à 48 h, ce qui s'avère trop long pour évaluer sur site l'efficacité des traitements et adapter les stratégies de traitement (nature et dose des produits désinfectants). Devant ces difficultés, les traitements chimiques préventifs sont le plus souvent mis en œuvre suivant une base calendaire, et non au regard d'une évaluation des risques. Ceci ne permet pas toujours d'empêcher l'émergence d'épisodes de contamination et conduit les exploitants et les traiteurs d'eau à augmenter leurs doses de désinfectants, provoquant de ce fait une augmentation conjointe de la formation de sous-produits de désinfection (composés organohalogénés en particulier), rejetés dans le milieu naturel dans les eaux de purge des circuits.

Disposer d'un outil rapide et sensible d'évaluation du risque de prolifération bactérienne présente donc un intérêt ma- jeur dans ce contexte. La détection des bactéries pathogènes constituerait évidemment la clé du problème, mais les travaux récents soulignent que les biocapteurs présentent encore des performances irrégulières et ne peuvent pas encore fournir de réponses rapides [8]. Depuis quelques années des capteurs de biofilm se sont donc développés pour tenter d'obtenir une détection instantanée, simple et non destructive, d'autres paramètres caractéristiques de ces matrices (densité, porosité, épaisseur, conductivité électrique, absorption de lumière, ...). Des méthodes basées sur des principes divers ont ainsi vu le jour. Parmi ceux-ci les capteurs à détection électrochimique se taillent une place de choix [9 à 12].

Mais l'utilisation industrielle de ces capteurs souffre encore de quelques lacunes (en particulier pour l'application à des eaux de conductivité faibles ou moyennes) et quelques verrous technologiques restent encore à lever.

Un système de détection par voie électrochimique, permettant de suivre le transport de matière à travers la couche poreuse du biofilm, a été développé au sein de nos équipes [13]. Utilisant une électrode à disque tournant, il permet de déterminer l'épaisseur moyenne des biofilms formés sur la surface d'une électrode placée dans un circuit, à l'échelle pilote ou en milieu industriel. Les biofilms se caractérisent en effet par une structure de porosité très élevée, avec une teneur en eau généralement supérieure à $95 \%$. En ce qui concerne le transport de matière, ils se comportent comme une couche poreuse inerte et ils peuvent être considérés comme une couche d'eau stagnante à la surface du matériau [9]. Cette couche poreuse d'épaisseur $\delta_{f}$, constitue une barrière de diffusion et l'augmentation de l'épaisseur provoquera une diminution du flux de matière. La formation du biofilm à la surface d'une électrode pourra donc être suivie en déterminant l'évolution du courant de diffusion.

\section{Méthodes expérimentales}

\section{Le système électrochimique}

Le système électrochimique choisi pour réaliser ce suivi est une cellule à trois électrodes : électrode de travail à disque tournant, électrode de référence au calomel et contre-électrode. Il permet la mesure ampérométrique du flux de matière d'une espèce électroactive (traceur électrochimique) à la 
surface de l'électrode, c'est-à-dire à l'interface métal/biofilm.

Des électrodes de platine de $2 \mathrm{~mm}$ de diamètre (Radiometer Analytical) sont utilisées pour cette application. Immergées dans des circuits d'eau, à l'échelle pilote ou industrielle, ces électrodes dont la surface va se recouvrir de biofilm serviront de capteur pour les mesures d'épaisseur.

Le traceur électrochimique employé dans la cellule de mesure est le ferricyanure de potassium. Le contrôleur de vitesse de l'électrode à disque tournant est une unité CTV 101 de Radiometer Analytical et la détermination s'effectue avec un potentiostat SI 1280B de Solartron Analytical. La commande et l'acquisition des données sont réalisées grâce au logiciel CorrWare de Solartron Analytical.

\section{Les réacteurs}

Afin de simuler les conditions présentes dans les circuits de refroidissement ou les circuits d'eaux chaudes, divers réacteurs ont été conçus. Alimentés en continu par des eaux de surface ou par des eaux industrielles, ces réacteurs permettent d'une part d'implanter des électrodes pour la mesure de l'épaisseur des biofilms, et d'autre part des coupons (ou des billes de verre) permettant de récupérer le biofilm à des fins analytiques (analyses chimiques ou bactériologiques).

Avant le début de chaque phase de colonisation, la surface en platine de l'ensemble des électrodes de mesure est soigneusement nettoyée. Le courant mesuré pour cette surface propre correspondra alors à une épaisseur nulle de biofilm.

\section{Réacteurs de laboratoire}

Il s'agit de 2 réacteurs tubulaires (tubes en verre de $50 \mathrm{~cm}$ de long et $3 \mathrm{~cm}$ de diamètre), placés en série (Fig. 1). Le premier réacteur contient 4 ou 5 électrodes dont la surface en platine de $3,1 \mathrm{~mm}^{2}$ (diamètre de $2 \mathrm{~mm}$ ) sera colonisée par un biofilm. Leurs surfaces réactives sont affleurantes à la surface interne du réacteur. Le second réacteur contient des billes de verre de $5 \mathrm{~mm}$ de diamètre. Le système, placé dans une enceinte thermostatée à $37^{\circ} \mathrm{C}$, est alimenté en eau de surface par une pompe péristaltique à un débit de $0,5 \mathrm{~L} / \mathrm{h}$. Une pompe de recirculation permet d'assurer un régime hydraulique turbulent dans l'ensemble du circuit.

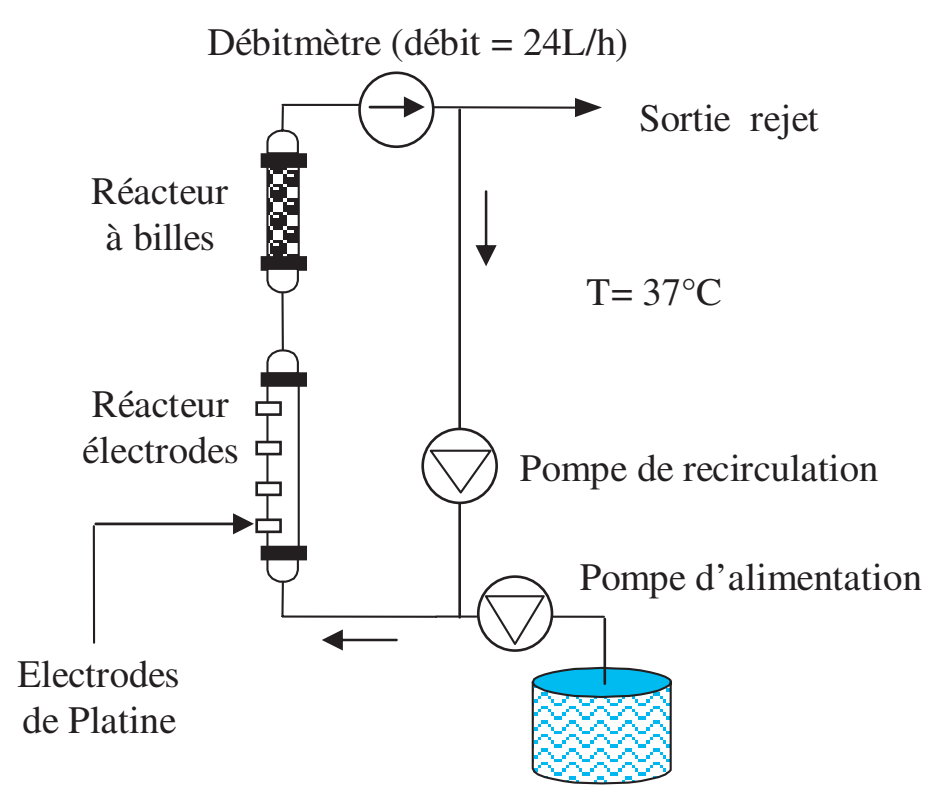

Alimentation en eaux $(0.4 \mathrm{~L} / \mathrm{h})$

Fig. 1. Schéma du réacteur de laboratoire alimenté en continu en eau naturelle et composé d'un réacteur tubulaire équipé d'électrodes de mesure et d'un réacteur à billes.

Fig. 1. Scheme of the laboratory incubator continuously fed out of natural wate. The incubator is composed of a tubular incubator equipped with measuring electrodes and of an incubator with glass balls.

Ce montage a été mis en œuvre pour étudier la fiabilité, la reproductibilité et la limite de détection de cette méthode, et pour examiner le potentiel de cette mesure à traduire les évolutions des biofilms sous l'effet des traitements.

\section{Dispositif expérimental utilisé sur site industriel}

Le capteur de biofilm développé en laboratoire a été validé sur site industriel dans les canalisations des circuits de refroidissement alimentant les tours aéroréfrigérantes de la Société d'Équipement de la Région Montpellieraine (SERM).

Le réacteur utilisé (Fig. 2) est placé en dérivation du circuit industriel (tours aéroréfrigérantes). Il est balayé à débit constant par l'eau du circuit. Comme précédemment, les électrodes de mesure, immergées dans l'eau du circuit, seront progressivement colonisées par un biofilm, au même titre que le matériau de la canalisation principale.

D'une longueur de $50 \mathrm{~cm}$ et d'un diamètre intérieur de $40 \mathrm{~mm}$, ce réacteur permet d'accueillir 4 électrodes, constituées d'un disque de platine de $5 \mathrm{~mm}$ de diamètre monté sur une tige en acier inoxydable enrobée d'une résine isolante (Fig. 2 à droite). 


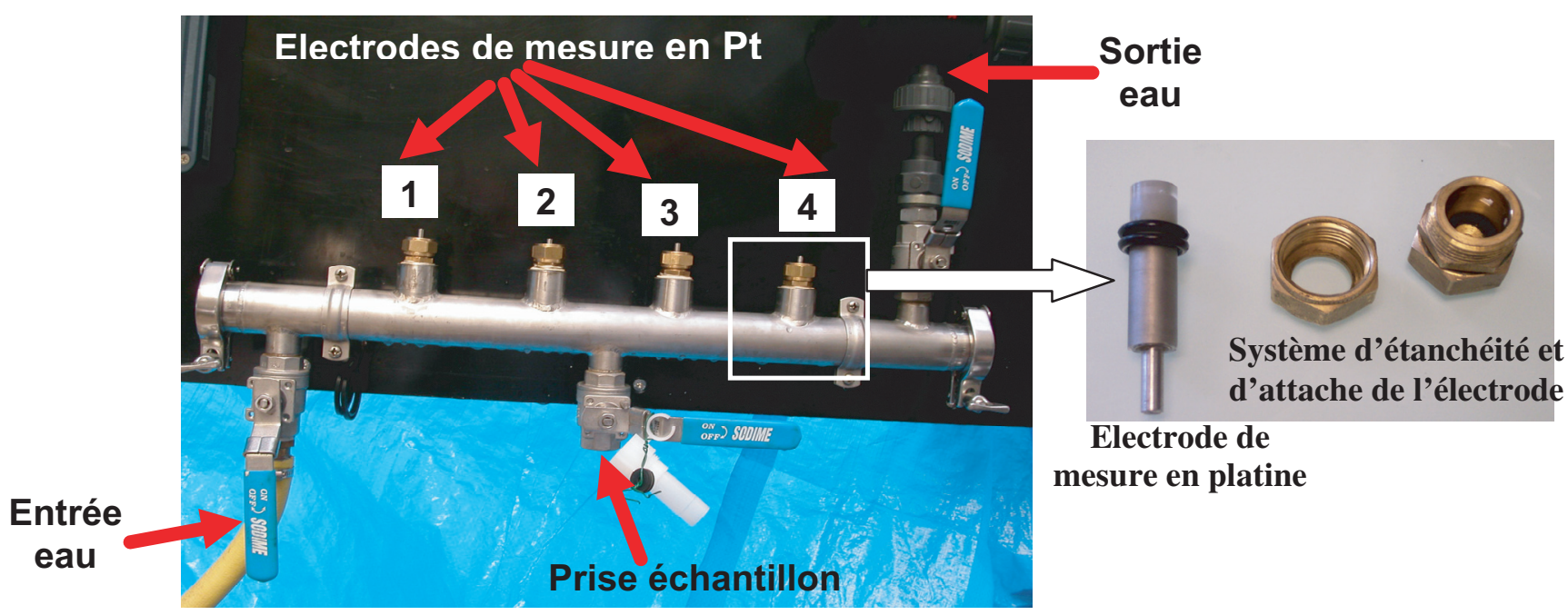

Fig. 2. Photographie du réacteur industriel servant de support aux électrodes de platine au sein du circuit de refroidissement du site de Montpellier.

Fig. 2. Industrial incubator used as a support for the platinum electrodes within the coolant circuit of the Montpellier site.

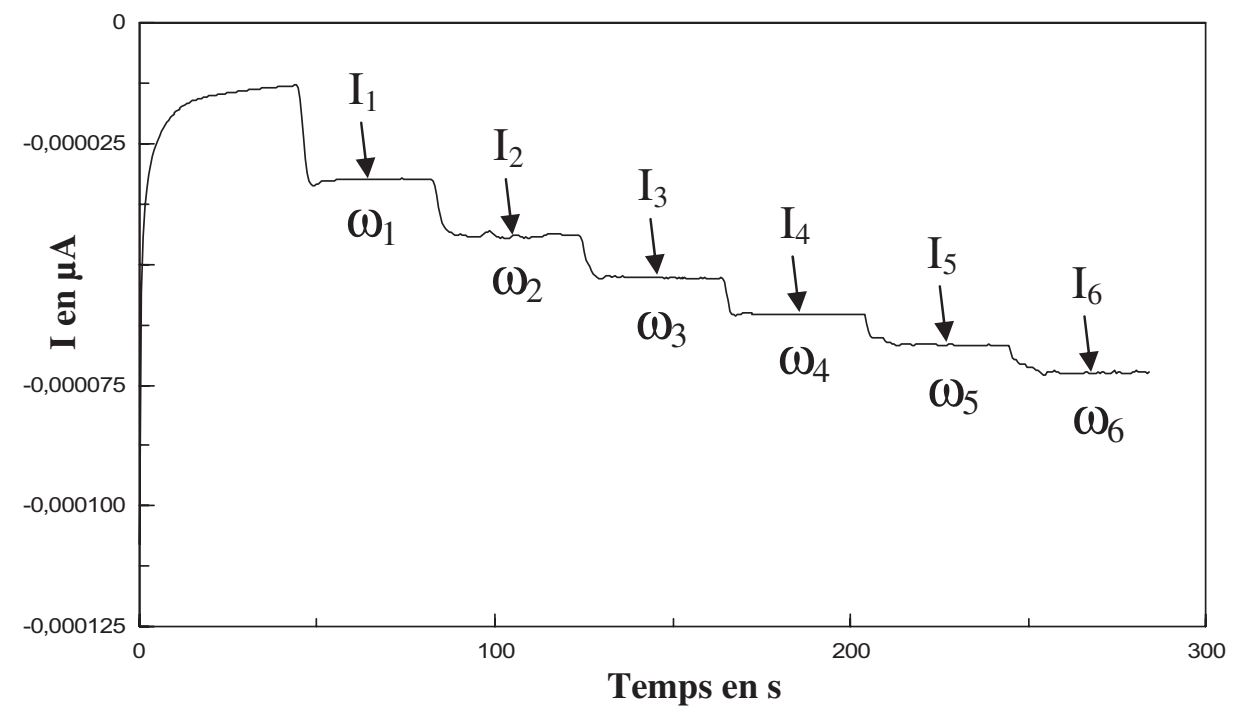

Fig. 3. Évolution du courant de diffusion I en fonction du temps pour des vitesses croissantes de rotation appliquées par l'électrode tournante.

Fig. 3. Evolution of the diffusion current I as a function of time for increasing speeds of rotation applied by the revolving electrode.

\section{Développement de l'outil électrochimique de détermination de l'épaisseur du biofilm}

\section{Suivis du courant de diffusion}

Au cours de nos travaux visant à caractériser le biofilm, le signal étudié est le courant correspondant à une réaction électrochimique entièrement limitée par le transport de matière et produite à l'interface métal/biofilm. Plus précisément, il s'agit en fait d'analyser l'écart entre le courant mesuré (en présence de biofilm), et le courant de Levich (en ab- sence de biofilm). Ainsi la technique expérimentale correspondante consiste à analyser la variation du courant avec la vitesse de rotation $\omega$ de l'électrode (Fig. 3).

Pour suivre la formation du biofilm au cours du temps, cette procédure sera appliquée pour différents temps d'immersion des électrodes de mesure et donc pour des âges différents de biofilm.

\section{Suivi de la formation du biofilm}

L'évolution temporelle de la croissance des biofilms d'eaux de rivière étudiés au cours 

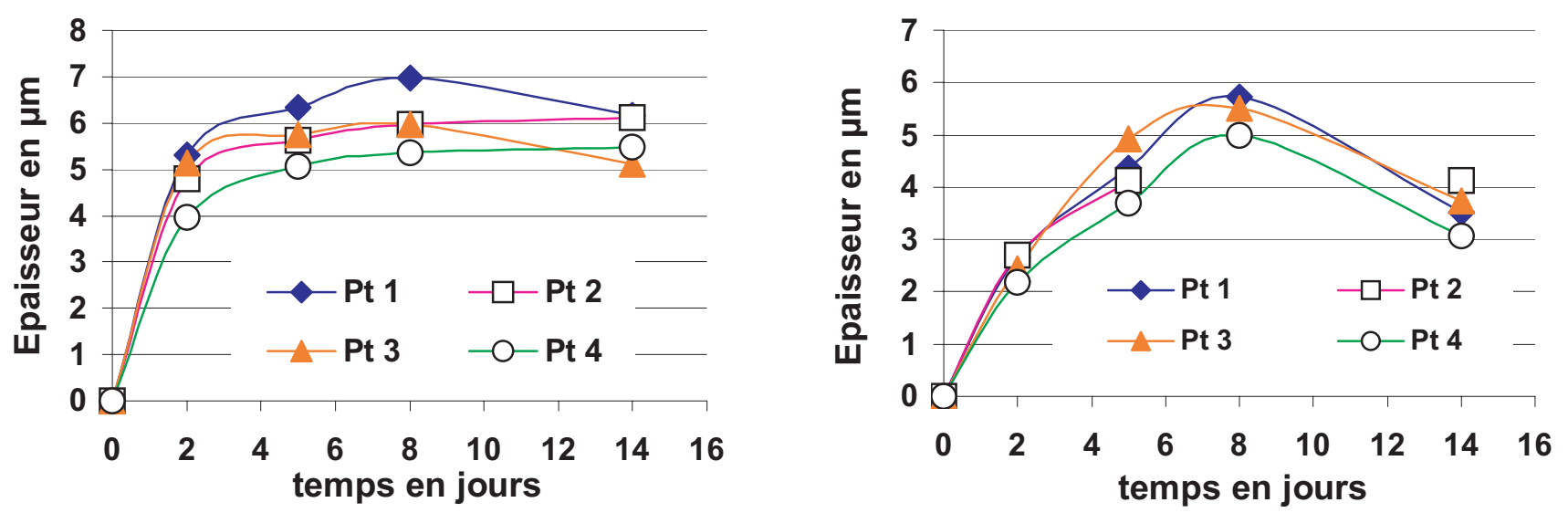

Fig. 4. Évolution de l'épaisseur à 300 tours/min d'un biofilm $A$ (à gauche) et $B$ (à droite) d' eau de rivière après 14 jours de colonisation (mesures effectuées sur 4 électrodes de platine : $\mathrm{Pt} 1$ à $\mathrm{Pt}$ 4).

Fig. 4. Thickness evolution at 300 turns/min of a biofilm A (on the left) and B (on the right) of river water after 14 days of colonization (measurements from 4 platinum electrodes: Pt 1 to Pt 4).

de ce travail sur des périodes de 10 à 20 jours montre, de manière générale, que l'épaisseur connaît une phase initiale de croissance dont la durée varie d'un biofilm à l'autre et qui peut s'étendre sur une période de 8 à 10 jours (Fig. 4).

Cette phase de croissance rapide est couramment admise par l'ensemble de la communauté scientifique [14]. Elle laisse place ensuite à des phases concomitantes d'adhésion/décrochage plus ou moins accentuées au cours desquelles la matrice du biofilm acquiert une plus grande maturité et tend à se stabiliser.

Après 14 jours d'immersion, l'épaisseur des 2 biofilms présentée sur la figure 4 atteint une valeur de 4 à $6 \mu \mathrm{m}$. Toutefois, ces deux biofilms présentent des profils de croissance différents : dans le premier cas (biofilm A), l'épaisseur maximale (5 à $7 \mu \mathrm{m}$ ) est atteinte dès le deuxième jour $\mathrm{d}^{\prime}$ immersion puis reste quasi-stable jusqu'à la fin de la phase de colonisation, alors que dans le second cas (biofilm B), l'épaisseur maximale (5 à $6 \mu \mathrm{m}$ ) est atteinte en 8 jours.

Pour l'ensemble des biofilms étudiés, on retrouve l'une ou l'autre de ces allures générales de cinétique de croissance, qui sont en accord avec les données de la littérature [15].

D'autre part, il est intéressant de noter que les mesures d'épaisseur réalisées en parallèle sur plusieurs électrodes de platine, prélevées à des temps d'immersion identiques, ne traduisent aucune différence significative.

Cette reproductibilité laisse supposer que si la formation d'un biofilm est effectivement hétérogène à l'échelle microscopique, cette hétérogénéité n'est plus un facteur appréciable à l'échelle macroscopique, et en particulier sur un disque de $2 \mathrm{~mm}$ de diamètre.

\section{Elasticité du biofilm}

La mesure des épaisseurs du biofilm avec le système à électrode tournante qui permet d'appliquer différentes vitesses de rotation nous a permis de mettre en évidence un comportement élastique du biofilm.

Ainsi la figure 5 présente l'évolution de l'épaisseur d'un biofilm formé sur une électrode de platine placée dans un réacteur alimenté en eau de rivière (le Clain) pendant 12 jours. A chaque temps d'immersion, l'épaisseur est déterminée pour différentes vitesses variant de 50 à 300 tours/min.

Pour un biofilm «jeune» (moins de 7 jours), ces courbes montrent que l'épaisseur mesurée est d'autant plus faible que la vitesse de rotation est élevée, caractérisant ainsi un biofilm élastique. Pour des biofilms plus âgés l'épaisseur mesurée devient indépendante de la vitesse de rotation, traduisant une faible élasticité et donc une certaine rigidité de la structure.

Cette notion d'élasticité, mise en évidence dans nos conditions expérimentales, s'explique par les propriétés structurales du biofilm évoquées dans la littérature [16], où le caractère poreux du biofilm résulte de l'organisation morphologique autour d'amas cellulaires, qui, sous la contrainte, se tassent sur le support entraînant alors une diminution globale de l'épaisseur du biofilm. Ce phénomène peut également être attribué à la forte minéralisation de certains biofilms, telle celle décrite par Hiernaux [5]. 


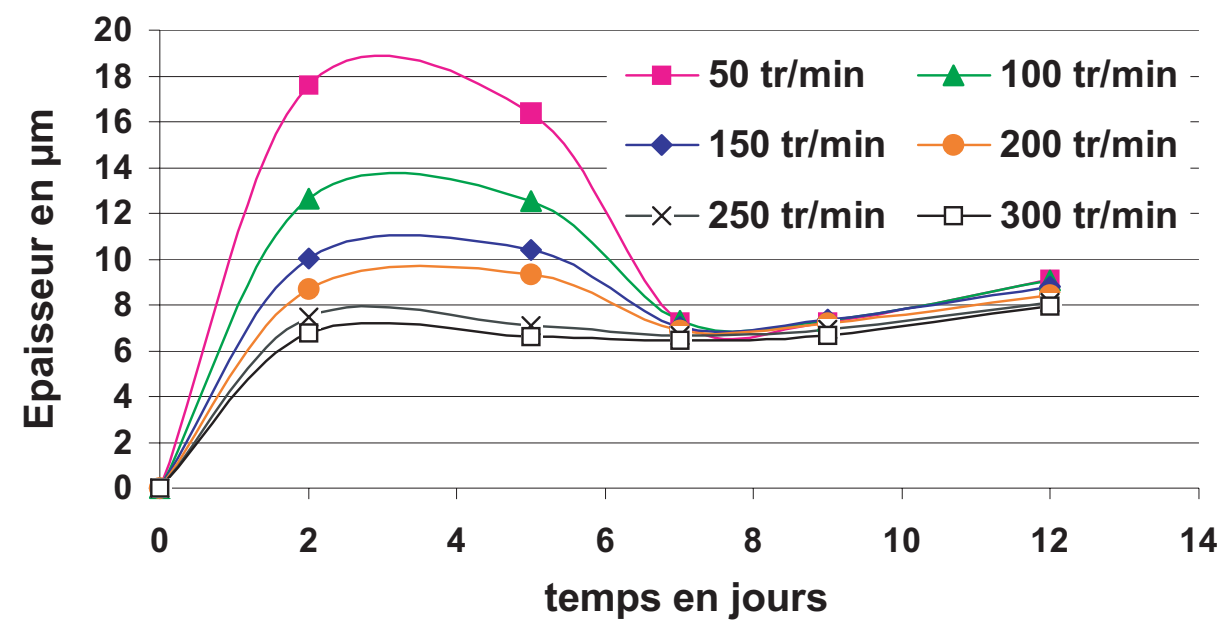

Fig. 5. Évolution de l'épaisseur d'un biofilm en fonction de la vitesse de rotation de l'électrode pour une gamme allant de 50 à 300 tours/min durant 12 jours de colonisation.

Fig. 5. Thickness evolution of a biofilm as a function of the rotation speed of the electrode within a range from 50 to 300 turns/min during 12 days of colonization.

\section{Application au suivi de l'efficacité des traitements des circuits}

\section{Étude comparative en laboratoire de I'impact d'une gamme de dispersants}

Afin d'évaluer au mieux l'aptitude du procédé développé à suivre l'évolution de l'épaisseur lors de phases de traitement, plusieurs produits à propriétés dispersantes de la gamme Henkel Concorde ont fait l'objet d'essais sur les unités pilote de laboratoire. Ces produits ont été appliqués sur des biofilms matures de 10 jours formés sur des électrodes de platine mais aussi sur des billes de verre (d'un diamètre de $4 \mathrm{~mm}$ ).

Outre les mesures d'épaisseurs, les analyses effectuées sur le biofilm sont les dénombrements bactériens totaux, la biomasse fixée étant récupérée sur les billes de verre par sonication.

Les produits dispersants testés et leur principe actif majoritaire sont les suivants :

- D1 : alkylamides d'acides gras,

- D2 : copolymère cationique,

- D3 : copolymère amphotère,

- D4 : copolymère acrylique.

La figure 6 présente l'évolution de l'épaisseur du biofilm de 10 jours, à 300 tours/min, sous l'action de ces différents produits à différents temps de contact (de 1 à 5 heures).

La mesure de l'épaisseur du biofilm grâce à l'outil électrochimique permet de distinguer des comportements différents des formulations dispersantes au cours du traitement. En effet, s'il est intéressant de constater une réduction importante de l'épaisseur du biofilm pour l'ensemble des dispersants testés, il faut également souligner que le produit D4 conduit à une réduction importante et immédiate de l'épaisseur du biofilm alors que les produits dispersants D1 et D2 ont un comportement quasi-similaire avec une diminution très significative de l'épaisseur mais seulement après 3 heures de temps de contact. Après 5 heures de temps de contact, les épaisseurs ont diminué respectivement de :

- $56 \%$ pour D2,

- $63 \%$ pour D1,

- $71 \%$ pour D3,

- $74 \%$ pour D4.

L'intérêt de cette mesure de l'épaisseur pour évaluer l'efficacité d'un traitement est confirmé par les résultats présentés sur la figure 7. Au cours de cet essai, nous avons suivi l'évolution de l'épaisseur grâce à la cellule à électrode tournante et la densité bactérienne cultivable, déterminée sur les billes de verre à la fin d'un traitement de 3 heures.

Deux concentrations de ce produit ont été testées en parallèle $(10 \mathrm{mg} / \mathrm{L}$ et $50 \mathrm{mg} / \mathrm{L})$ (Fig. 7). Pour les deux taux de traitement, la réduction de l'épaisseur du biofilm mesurée par le procédé à électrode tournante est en relation avec l'abattement de la densité bactérienne cultivable.

En effet, pour une concentration de $10 \mathrm{mg} / \mathrm{L}$, la mesure de l'épaisseur indique que le biofilm est partiellement éliminé avec une épaisseur qui diminue de 6 à $2 \mu \mathrm{m}$ en 5 heures. Cette efficacité partielle est confirmée par une diminution d'un facteur 10 de 


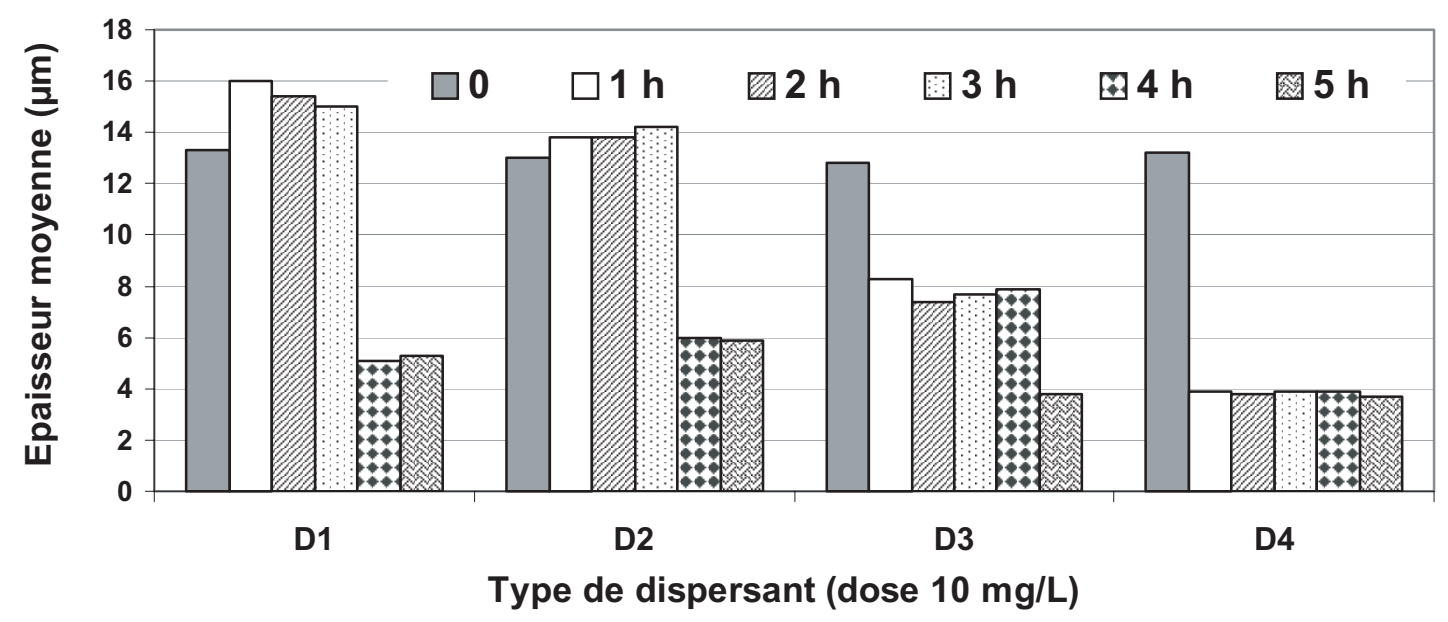

Fig. 6. Action des dispersants à $\mathbf{1 0} \mathrm{mg} / \mathrm{L}$ sur l'épaisseur d'un biofilm mature de $\mathbf{1 0}$ jours. Fig. 6. Action of the dispersing agents $(10 \mathrm{mg} / \mathrm{L})$ on a 10 days biofilm thickness.

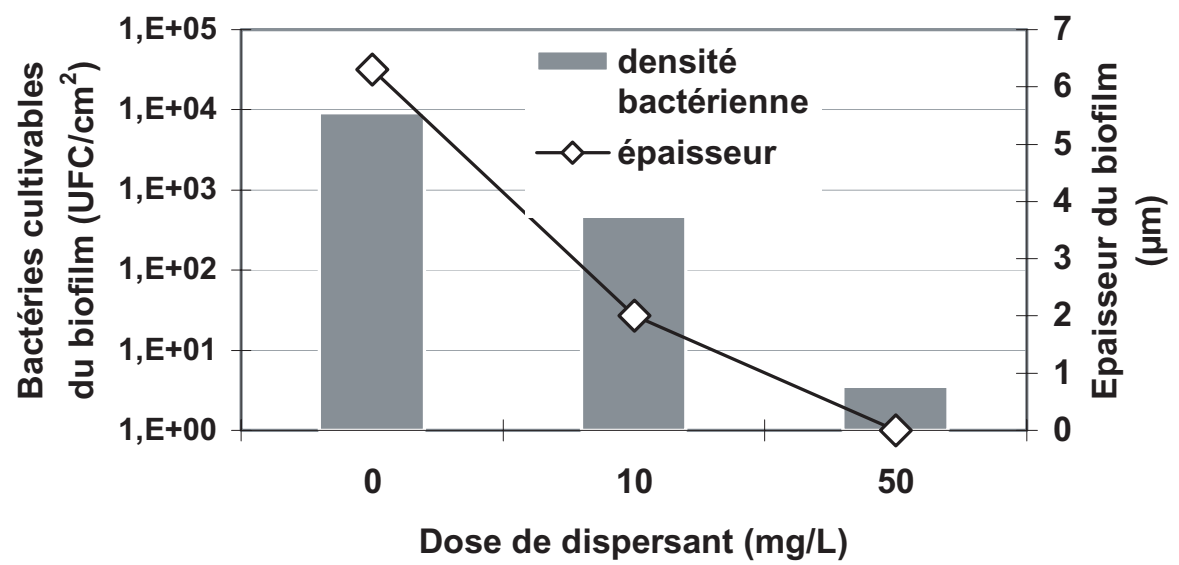

Fig. 7. Évolution de l'épaisseur et de la densité bactérienne totale d'un biofilm mature de 10 jours traité par une solution mère de dispersant à 10 et $50 \mathrm{mg} / \mathrm{L}$.

Fig. 7. Thickness evolution and total bacterial density of a 10 days mature biofilm treated with a mother solution of dispersant with 10 and $50 \mathrm{mg} / \mathrm{L}$ concentrations.

la densité bactérienne cultivable, avec la présence résiduelle d'environ $10^{3} \mathrm{UFC} / \mathrm{cm}^{2}$ de bactéries cultivables. Par contre, avec une concentration plus élevée (50 mg/L), on ne détecte plus aucun biofilm après 3 heures de contact et, parallèlement, l'abattement des bactéries cultivables est de plus de $3 \log$, leur teneur résiduelle à l'issue du traitement étant voisine de la limite de détection de la méthode.

\section{Essais sur site industriel}

Le potentiel du capteur pour suivre le développement du biofilm a été testé sur un site industriel à Montpellier par la société Henkel Concorde.

Dans ce cadre, des électrodes de mesure (paragraphe Dispositif expérimental utilisé sur site industriel) ont été disposées au sein des circuits de refroidissement d'une entreprise chargée de la distribution de chaleur et de froid de la ville de Montpellier.

$\mathrm{Au}$ cours de la campagne de mesure, un traitement en injections séquencées de biocide est appliqué tous les 7 jours par le contractant industriel pour prévenir la formation de biofilm et ainsi limiter les risques de prolifération des germes pathogènes opportunistes tels Legionella pneumophila.

Pour l'ensemble des électrodes placées sur le circuit, les évolutions sont similaires ; il a donc été représenté sur la figure 8 l'évolution de l'épaisseur moyenne, en fonction du temps d'immersion et de la concentration $d u$ biocide injecté.

Sur ce circuit, le produit biocide est injecté tous les 7 jours à une concentration de $40 \mathrm{mg} / \mathrm{L}$ et la figure 8 montre une diminution 


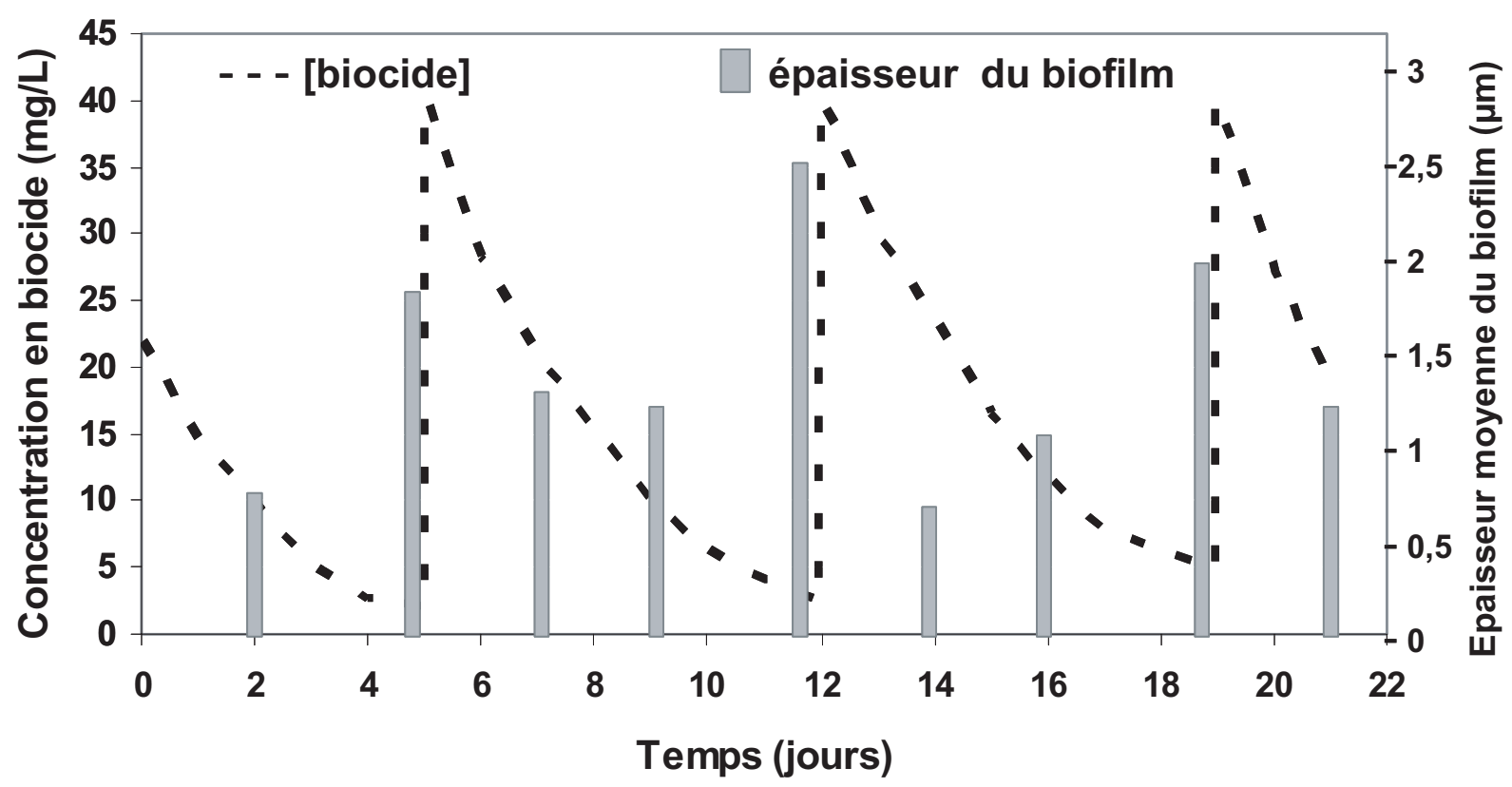

Fig. 8. Impact de traitements biocides séquencés sur l'évolution de l'épaisseur moyenne d'un biofilm mesurée sur 5 électrodes immergées dans un circuit industriel.

Fig. 8. Impact of biocides sequenced treatments on the evolution of an average biofilm thickness, measured on 5 electrodes immersed in an industrial circuit.

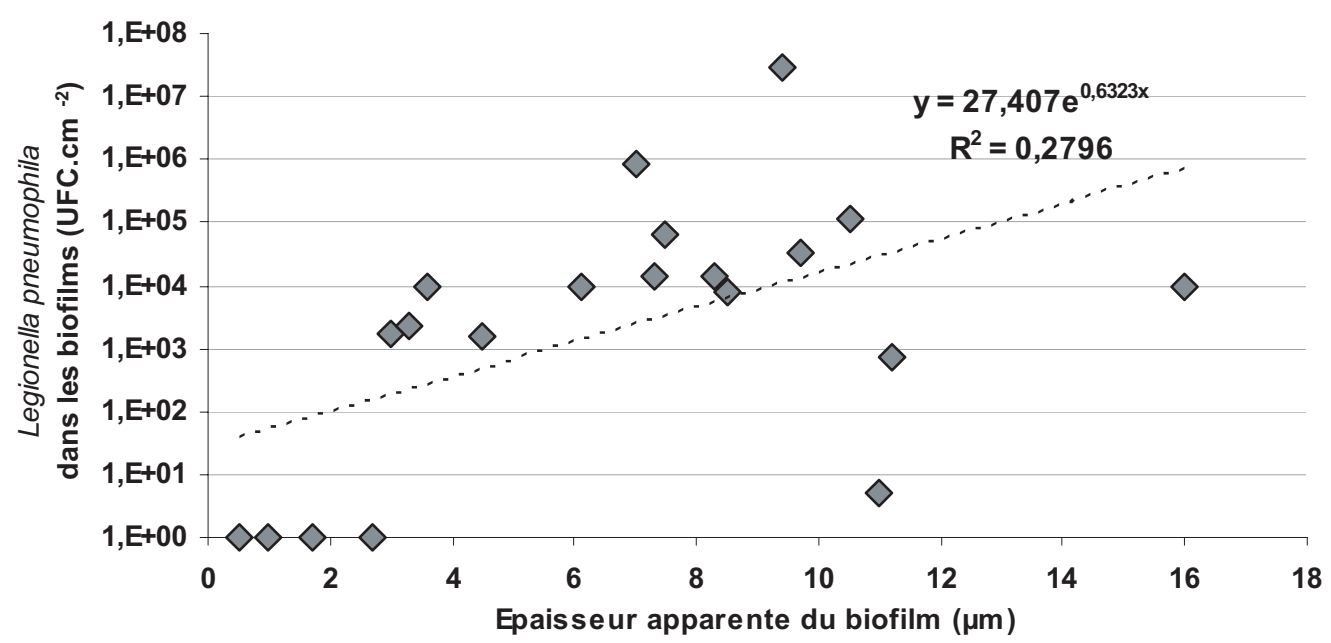

Fig. 9. Évolution de la teneur en Legionella pneumophila (en UFC/ $\mathrm{cm}^{2}$ ) en fonction de l'épaisseur moyenne des differents biofilms étudiés.

Fig. 9. Evolution of the Legionella pneumophila concentration (in UFC/ $\mathrm{cm}^{2}$ ) as a function of the average thickness, in different biofilms.

rapide de cette concentration dans le circuit avec un abattement de près de $50 \%$ après deux jours.

Si l'on examine les évolutions des épaisseurs de biofilm mesurées en différents points du circuit en fonction de la teneur résiduelle en produit de traitement, force est de constater que ces évolutions sont corrélées :

- chaque injection de biocide provoque une diminution rapide de l'épaisseur sur toutes les électrodes,
- mais la concentration résiduelle présente dans le circuit ne permet pas de poursuivre l'action destructrice du biofilm et l'épaisseur recommence à augmenter dès que la concentration en produit devient trop faible (ici inférieure à $20 \mathrm{mg} / \mathrm{L}$ environ).

Ce scénario en deux étapes se reproduit à l'identique à chaque séquence d'injection. Toutefois, même si le biofilm reprend sa croissance après avoir subi un impact transitoire aussitôt après l'injection, la stratégie de 


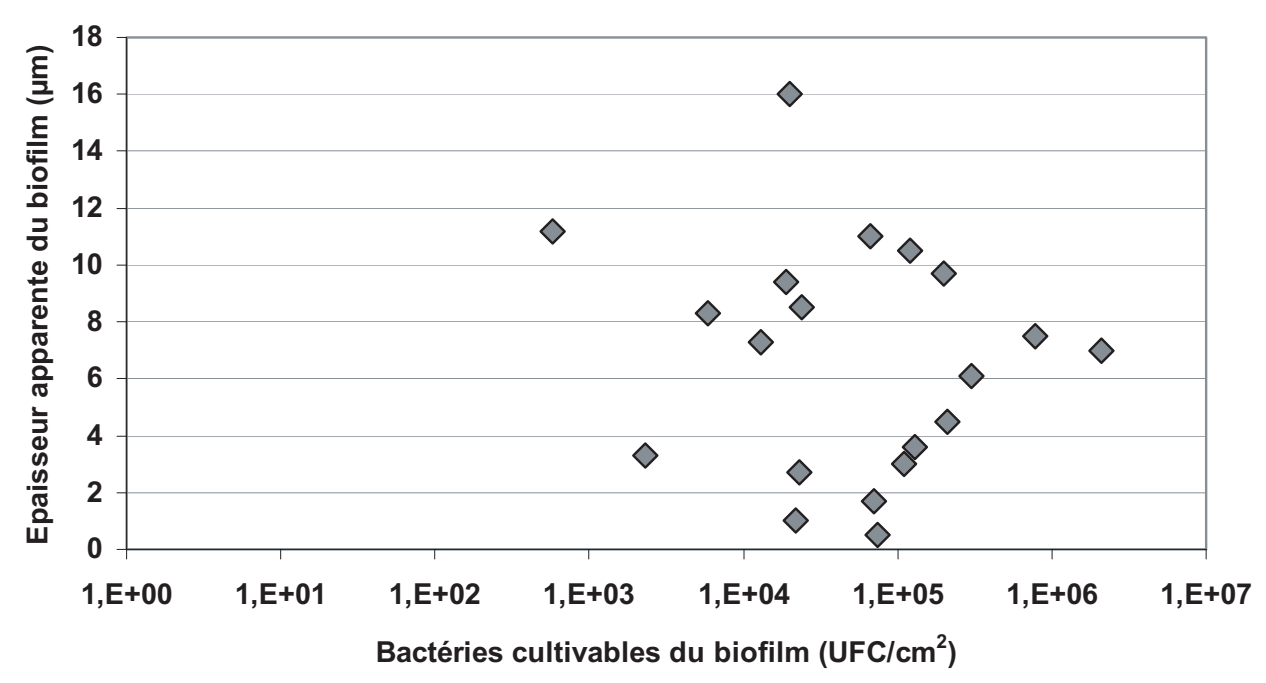

Fig. 10. Évolution de l'épaisseur moyenne des différents biofilms étudiés (en $\mu \mathrm{m}$ ) en fonction de la teneur en bactéries cultivables (en UFC/ $\mathrm{cm}^{2}$ ).

Fig. 10. Evolution of the average thickness of the different biofilms studied (in $\mu m$ ) as a function of viable bacteriaconcentration (in UFC/ $\mathrm{cm}^{2}$ ).

traitement mise en place et optimisée grâce au capteur permet de maîtriser l'épaisseur du biofilm à un seuil acceptable (inférieur à $3 \mu \mathrm{m}$ ) et assure donc une maîtrise du risque légionelle.

\section{Méthode d'évaluation du risque légionelle}

La validation de cet outil analytique de détermination de l'épaisseur d'un biofilm nécessite d'examiner les éventuelles relations entre le paramètre épaisseur mesuré et d'autres caractéristiques du biofilm, et en particulier les densités bactériennes fixées. Des expérimentations sur 30 eaux d'origines diverses (eaux de surface ou eaux souterraines de compositions variées) ont donc été menées en laboratoire sur des réacteurs identiques à celui présenté figure 1. Les biofilms formés à $37^{\circ} \mathrm{C}$ sont caractérisés après 10 jours d'alimentation en continu à une température de $37^{\circ} \mathrm{C}$.

Ainsi, les figures 9 et 10 montrent les teneurs en Legionella pneumophila et en bactéries cultivables dans les biofilms en fonction des épaisseurs moyennes déterminées sur ces mêmes biofilms.

À ce jour, les données de la bibliographie soulignent qu'il est difficile de relier fiablement la présence et la teneur en Legionella pneumophila avec tout autre paramètre expérimental relatif à l'eau ou au biofilm.

Toutefois, il est intéressant de constater sur la figure 9 que les biofilms peu épais (épaisseur $<3 \mu \mathrm{m}$ ) sont ceux dont les teneurs en légionelles sont les plus faibles (et même nulles dans nos conditions expérimentales). D'autre part, des teneurs élevées en légionelles $\left(10^{4}\right.$ à $\left.10^{7} \mathrm{UFC} / \mathrm{cm}^{2}\right)$ sont généralement détectées dans des biofilms plus épais (5 et $10 \mu \mathrm{m}$ ).

Mais chaque biofilm présente ses caractéristiques propres (composés organiques et minéraux, constituants bactériens, ...), liées pour partie à la composition des eaux d'alimentation et aux conditions de fonctionnement des circuits (température, régime hydraulique, matériau) et des corrélations directes entre 2 paramètres de caractérisation du biofilm semblent difficiles à concevoir. Ainsi, la figure 10 montre qu'il n'existe pas de réelle corrélation entre la densité bactérienne cultivable dans le biofilm et les épaisseurs mesurées. En effet, quelle que soit leur épaisseur, tous les biofilms présentent des teneurs importantes en bactéries cultivables sur milieu PCA (de $10^{4}$ à $10^{5} \mathrm{UFC} / \mathrm{cm}^{2}$ ). Et de façon générale, dans nos conditions expérimentales, les biofilms, qui présentaient des épaisseurs moyennes apparentes de 1 à $16 \mu \mathrm{m}$ contenaient des teneurs bactériennes cultivables comprises entre $10^{4}$ à $10^{6} \mathrm{UFC} / \mathrm{cm}^{2}$.

\section{Conclusion}

Le capteur électrochimique développé dans le cadre de ces travaux constitue un outil analytique précis, sensible et reproductible 
pour mesurer le transfert de matière au sein de la couche poreuse constituée par le biofilm présent à la surface d'un matériau. La grandeur mesurée est l'épaisseur moyenne de ce biofilm, et la mesure est non destructive, ce qui permet un suivi dans le temps sur un même capteur.

L'utilisation de la cellule à électrode tournante pour suivre l'évolution temporelle du biofilm au cours de traitements désinfectants (biocide, dispersant, biodispersant, ...) en laboratoire et sur site industriel confirme l'efficacité de ce type de capteur pour évaluer l'impact et l'efficacité des procédures visant à contrôler le biofilm au sein des réseaux.

Cet outil de détermination électrochimique de l'épaisseur d'un biofilm s'avère donc capable :

- de traduire l'impact plus ou moins marqué d'un traitement,

- de discriminer différents produits de traitement en fonction de leurs caractéristiques ou de leur concentration,

- de donner un signal significatif et reproductible corrélable avec les valeurs de densités bactériennes cultivables et en particulier de présenter une sensibilité suffisante pour indiquer une épaisseur nulle quand la densité bactérienne cultivable est proche de la limite de détection,

- de mettre en exergue les phénomènes de résistance aux désinfectants qui s'opèrent fréquemment dans les biofilms.

L'ensemble de ces essais expérimentaux positionne le capteur à électrode tournante comme un outil de surveillance pertinent pour le suivi des biofilms et l'optimisation des traitements physico-chimiques.

Le développement d'un capteur industriel découlant de ces travaux est en cours dans le cadre d'un partenariat entre les équipes CNRS UMR 6008 et UPR 15 et la société HENKEL Concorde.

\section{Remerciements}

Ce travail a été soutenu financièrement par la région Poitou-Charentes et par le programme «Eaux » du XII ${ }^{e}$ contrat de Plan Etat/Région.

\section{Références}

[1] C.W. Keevil, Pathogens and metabolites associated with biofilms, Biofilms in the aquatic environment, edited by C.W. Keevil, A. Godfree, D. Holt, C. Dow, Royal Society of Chemistry, Cambridge, UK (1999) 145-152

[2] P.H. Nielsen, A. Jahn, R. Palmgren, Water. Sci. Technol. 36 (1997) 11-19

[3] A. Jahn, P.H. Nielsen, Water Sci. Technol. 37 (1998) 17-24

[4] H.C. Flemming, J. Wingender, T. Griebe, C. Mayer, Physico-chemical properties of biofilms. Biofilms: Recent advances in their study and control, edited by L.V. Evans, Harwood Academic Publishers, Amsterdam, The Netherlands (2000) 19-34

[5] P. Hiernaux, Contribution de la fraction minérale des eaux au développement et à la structure des biofilms : Apport des méthodes microscopiques et spectroscopiques, Thèse, Université de Poitiers, 2005

[6] AFNOR NF T90-431, Qualité de l'eau Recherche et dénombrement de Legionella spp et de Legionella pneumophila Méthode par ensemencement direct et après concentration par filtration sur membrane ou centrifugation, 2003

[7] AFNOR XP T90-471, Qualité de l'eau Détection et quantification des Legionella et/ou Legionella pneumophila par concentration et amplification génique par réaction de polymérisation en chaîne (PCR), 2006

[8] O. Lazcka, F.J. Del Campo, F.X. Muňoz, Biosensors Bioelectr. 22 (2007) 1205-1217

[9] D. Herbert-Guillou, B. Tribollet, D. Festy, L. Kiené, Electrochem. Acta 45 (1999) 1067-1075

[10] A. Mollica, Simple electrochemical sensors for biofilm and MIC monitoring, Meeting of task 5, Microbially influenced corrosion of industrial materials - Biocorrosion network, Venise, Italy (2000)

[11] M.C Bruijs, L.P. Venhuis, H.A. Jenner, G.J. Licina, D. Daniels, PowerPlant Chem. (2001) 3-7

[12] A. Bressel, J.W. Schultze, W. Khan, G.M. Wolfaardt, H.P. Rohns, R. Irmscher, M.J. Schöning, Electrochem. Acta 48 (2003) 3363-3372

[13] C. Forêt, G. Chaussec, N. Merlet, S. Martemianov, B. Tribollet, L'Eau, l'Industrie, les Nuisances 299 (2007) 41-45

[14] J.W. Costerton, Inter. J. Antimicr. Agents 11 (1999) 217-221

[15] J.D. Bryers, Biofilm formation and persistence, in Biofilms II Process analyses and applications, edited by J.D. Bryers, John Wiley \& Sons, New York (2000) 45-88

[16] J.W. Costerton, Z. Lewandowski, D. Debeer, D.E. Caldwell, D.R. Korber, G. James, J. Microbiol. (1994) 2137-2142 